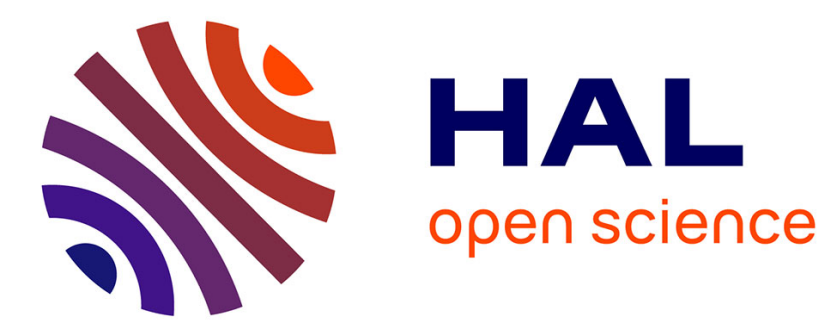

\title{
Strange Expectations and Simultaneous Cores
}

Marko Thiel, Nathan Williams

\section{To cite this version:}

Marko Thiel, Nathan Williams. Strange Expectations and Simultaneous Cores. 28-th International Conference on Formal Power Series and Algebraic Combinatorics, Simon Fraser University, Jul 2016, Vancouver, Canada. 10.46298/dmtcs.6364 . hal-02166326

\section{HAL Id: hal-02166326 https://hal.science/hal-02166326}

Submitted on 26 Jun 2019

HAL is a multi-disciplinary open access archive for the deposit and dissemination of scientific research documents, whether they are published or not. The documents may come from teaching and research institutions in France or abroad, or from public or private research centers.
L'archive ouverte pluridisciplinaire HAL, est destinée au dépôt et à la diffusion de documents scientifiques de niveau recherche, publiés ou non, émanant des établissements d'enseignement et de recherche français ou étrangers, des laboratoires publics ou privés. 


\title{
Strange Expectations and Simultaneous Cores
}

\author{
Marko Thiel听 and Nathan Williams" \\ ${ }^{1}$ Institut für Mathematik, Universität Zürich, Zürich, Switzerland \\ ${ }^{2}$ LaCIM, Université de Québec à Montéal, Montréal (Québec), Canada
}

\begin{abstract}
Let $\operatorname{gcd}(a, b)=1$. J. Olsson and D. Stanton proved that the maximum number of boxes in a simultaneous $(a, b)$-core is $\frac{\left(a^{2}-1\right)\left(b^{2}-1\right)}{24}$, and showed that this maximum is achieved by a unique core. P. Johnson combined Ehrhart theory with the polynomial method to prove D. Armstrong's conjecture that the expected number of boxes in a simultaneous $(a, b)$-core is $\frac{(a-1)(b-1)(a+b+1)}{24}$. We apply P. Johnson's method to compute the variance and third moment. By extending the definitions of "simultaneous cores" and "number of boxes" to affine Weyl groups, we give uniform generalizations of these formulae to simply-laced affine types. We further explain the appearance of the number 24 using the "strange formula" of H. Freudenthal and H. de Vries.
\end{abstract}

Résumé. Soit $\operatorname{gcd}(a, b)=1$. J. Olsson et D. Stanton ont prouvé que le nombre maximum de cases dans un $(a, b)$ core simultané est $\frac{\left(a^{2}-1\right)\left(b^{2}-1\right)}{24}$, et que ce maximum est atteint par un core unique. P. Johnson a combiné la théorie Ehrhart avec la méthode polynomiale afin de prouver une conjecture de D. Armstrong avançant que le nombre de cases dans un $(a, b)$-core simultané est $\frac{(a-1)(b-1)(a+b+1)}{24}$. Nous appliquons cette méthode de P. Johnson pour calculer la variance et le troisième moment. En étendant les définitions de "core simultanés" et "nombre de boîtes" aux groupes de Weyl affines, nous arrivons à des généralisations uniformes de ces formules pour tout type affine simplement-lacé. Nous expliquons l'apparition du nombre 24 en utilisant la "formule étrange" de H. Freudenthal et H. de Vries.

Keywords. Partition, core, Ehrhart theory, affine Weyl group

\section{Introduction}

\subsection{Motivation: Simultaneous Cores}

An $a$-core is an integer partition with no hook-length divisible by $a$. As a first example, observe that the 2-cores are exactly those partitions of staircase shape. According to the notes in G. James and A. Kerber [JK81], cores were originally developed by T. Nakayama in his study of the modular representation theory of the symmetric group [Nak40]. For $\lambda$ a partition of $k$, we write size $(\lambda):=k$. Let core $(a)$ be the set of all $a$-cores, and define core ${ }_{k}(a):=\{\lambda \in \operatorname{core}(a): \operatorname{size}(\lambda)=k\}$. The following identity relating integer partitions and $a$-cores is a fun exercise using the abacus:

\footnotetext{
†Email: marko.thielemath.uzh.ch.

‡Email: nathan.f.williams@gmail.com.
} 
Theorem 1.1 (Generating function for size on $\operatorname{core}(a)$; [JK81, GKS90])

$$
\sum_{k=0}^{\infty}\left|\operatorname{core}_{k}(a)\right| q^{k}=\prod_{i=1}^{\infty} \frac{\left(1-q^{a i}\right)^{a}}{1-q^{i}} .
$$

An $(a, b)$-core is a partition that is both an $a$-core and a $b$-core. We denote the set of $(a, b)$-cores by core $(a, b)$. When $a$ and $b$ are coprime, J. Anderson proved that there are only finitely many $(a, b)$-cores.

Theorem 1.2 (Number of simultaneous $(a, b)$-cores; J. Anderson [And02]) For $\operatorname{gcd}(a, b)=1$,

$$
|\operatorname{core}(a, b)|=\frac{1}{a+b}\left(\begin{array}{c}
a+b \\
b
\end{array}\right) .
$$

In part due to their connection with rational Dyck paths (and hence diagonal harmonics and the zeta map) and rational Catalan combinatorics, cores have recently attracted attention. Furthermore, the study of simultaneous cores has now transcended these original motivations, and they have become combinatorial objects worthy of study in their own right. In this direction, there are two main results on the statistic size.

Theorem 1.3 (Maximum size of an $(a, b)$-core; J. Olsson and D. Stanton [OS07]) For $\operatorname{gcd}(a, b)=1$,

$$
\max _{\lambda \in \operatorname{core}(a, b)}(\operatorname{size}(\lambda))=\frac{\left(a^{2}-1\right)\left(b^{2}-1\right)}{24} .
$$

This maximum is attained by a unique $(a, b)$-core.

A stronger statement is actually true: J. Vandehey proved that the diagram of this unique $(a, b)$-core maximizing size contains the diagrams of all other $(a, b)$-cores [Van08, Fay11] [OS07, Remark 4.11].

D. Armstrong conjectured the following attractive formula [Arm15a, AHJ14], which was proven for $b=a+1$ by R. Stanley and F. Zanello [SZ13]; for $b=m a+1$ by A. Aggarwal [Agg14]; and in full generality by P. Johnson [Joh15].

Theorem 1.4 (Expected size of an $(a, b)$-core; P. Johnson [ [.Joh15]) For $\operatorname{gcd}(a, b)=1$,

$$
\underset{\lambda \in \operatorname{core}(a, b)}{\mathbb{E}}(\operatorname{size}(\lambda))=\frac{(a-1)(b-1)(a+b+1)}{24} .
$$

Our first new result is to extend P. Johnson's technique to compute the variance of core $(a, b)$.

Theorem 1.5 (Variance of size on $(a, b)$-cores) For $\operatorname{gcd}(a, b)=1$,

$$
\underset{\lambda \in \operatorname{core}(a, b)}{\mathbb{V}}(\operatorname{size}(\lambda))=\frac{a b(a-1)(b-1)(a+b)(a+b+1)}{1440} .
$$

We also compute the third moment, which was conjectured by D. Armstrong in 2013 [Arm15b].

Theorem 1.6 (Third moment of size on $(a, b)$-cores) For $\operatorname{gcd}(a, b)=1$, let $\mu:=\underset{\lambda \in \operatorname{core}(a, b)}{\mathbb{E}}(\operatorname{size}(\lambda))$. Then

$$
\frac{1}{|\operatorname{core}(a, b)|} \sum_{\lambda \in \operatorname{core}(a, b)}(\operatorname{size}(\lambda)-\mu)^{3}=\frac{a b(a-1)(b-1)(a+b)(a+b+1)\left(2 a^{2} b-3 a^{2}+2 a b^{2}-3 a b-3 b^{2}-3\right)}{60480} .
$$




\subsection{Simply-Laced Generalizations}

The main purpose of this abstract is to give generalizations of Theorems 1.1 to 1.5 for all simply-laced types. This simply-laced requirement arises from a simplification that only happens in those types.

To this end, we fix the following notation, which is fully reviewed in Section 2 . Let $\Phi$ be an irreducible crystallographic root system of rank $n$ with ambient space $V$ and Weyl group $W$. Let $\widetilde{\Phi}$ be the set of affine roots and denote the affine Weyl group by $\widetilde{W}$. Choose a set of simple roots $\Delta$ for $\Phi$ and let $\Phi^{+}$ be the corresponding set of positive roots. Say $\Phi$ has exponents $e_{1} \leq e_{2} \leq \cdots \leq e_{n}$, Coxeter number $h:=e_{n}+1$, and dual Coxeter number $g$. Theorems 1.1 to 1.5 will be recovered in this notation by specializing to type $A_{a-1}$, in which case $n=a-1, h=a$ and $W=\widetilde{\mathfrak{S}}_{a}$.

A useful analogue of cores for $\widetilde{W}$ turns out to be the coroot lattice $\check{Q}$, which we emphasize with the notation $\operatorname{core}(\widetilde{W}):=\check{Q}$. In Section 3, we recall how this definition recovers $a$-cores when $\widetilde{W}=\widetilde{\mathfrak{S}}_{a}$. In order to generalize Theorems 1.1 and 1.3 to 1.5 , we require a notion of the statistic size, defined combinatorially for $\widetilde{\mathfrak{S}}_{a}$ using the Ferrers diagram of a core. For the purposes of the introduction, we pull this out of a hat (but see Definition 6.1 and Example 6.3): for any point $x \in V$ - and in particular for any point in the coroot lattice $\dot{Q}$-let $\operatorname{size}(x):=\frac{g}{2}\|x\|^{2}-\langle x, \rho\rangle$, where $\rho:=\frac{1}{2} \sum_{\alpha \in \Phi^{+}} \alpha$ is half the sum of all positive roots. The statistic size on core $\left(\widetilde{\mathfrak{S}}_{a}\right)$ recovers the number of boxes in the corresponding core in core $(a)$ Definition 6.4 and Proposition 6.2.

Define $\operatorname{core}_{k}(\widetilde{W}):=\{\lambda \in \operatorname{core}(\widetilde{W}): \operatorname{size}(\lambda)=k\}$. By specializing his character formula at a primitive $h$ th root of unity, I. G. Macdonald uniformly generalized Theorem 1.1 to all simply-laced types.

Theorem 1.7 ([Mac71, Theorem 8.16]) For $\widetilde{W}$ the affine Weyl group of a simply-laced irreducible crystallographic root system $\Phi$ with Weyl group $W$, let $f(q)$ be the characteristic polynomial of a Coxeter element in $W$ (in the reflection representation). Then ${ }^{(i)}$

$$
\sum_{k=0}^{\infty}\left|\operatorname{core}_{k}(\widetilde{W})\right| q^{k}=\prod_{i=1}^{\infty}\left(f\left(q^{i}\right)\left(1-q^{h i}\right)^{n}\right) .
$$

Having generalized the notion of a core and the statistic size, we still require a definition of simultaneous cores. In Section 4, for any positive integer $b$ that is relatively prime to $h$, we write $b=t h+r$ with $0<r<h$ and define the Sommers region

$$
\mathcal{S}_{\Phi}(b):=\left\{x \in V:\langle x, \alpha\rangle \geq-t \text { for all } \alpha \in \Phi_{r} \text { and }\langle x, \alpha\rangle \leq t+1 \text { for all } \alpha \in \Phi_{h-r}\right\} .
$$

This is the region in $V$ bounded by all the affine hyperplanes corresponding to affine roots of height $b$. We define the generalisation of simultaneous cores for an affine Weyl group $\widetilde{W}$ as Definition 5.2

$$
\operatorname{core}(\widetilde{W}, b):=\mathcal{S}_{\Phi}(b) \cap \operatorname{core}(\widetilde{W})
$$

M. Haiman has uniformly proven (for all affine Weyl groups) the following generalization of Theorem 1.2 [Hai94], which we state in terms of $\mathcal{S}_{\Phi}(b)$ using a result of E. Sommers [Som05, Theorem 5.7]. We remark that R. Suter has also observed essentially the same formula type-by-type [Sut98].

(i) The last equality in [Mac71 Theorem 8.16] appears to have a small typo. 
Theorem 1.8 (Number of $(\widetilde{W}, b)$-cores; M. Haiman [Hai94]) For $\operatorname{gcd}(h, b)=1$,

$$
|\operatorname{core}(\widetilde{W}, b)|=\frac{1}{|W|} \prod_{i=1}^{n}\left(b+e_{i}\right) .
$$

We now state generalizations of Theorems 1.3 to 1.5

Theorem 1.9 (Maximum size of a $(\widetilde{W}, b)$-core) For $\widetilde{W}$ a simply-laced affine Weyl $\operatorname{group}, \operatorname{gcd}(h, b)=1$,

$$
\max _{\lambda \in \operatorname{core}(\widetilde{W}, b)}(\operatorname{size}(\lambda))=\frac{n\left(b^{2}-1\right)(h+1)}{24} .
$$

This maximum is attained by a unique $\lambda \in \operatorname{core}(\widetilde{W}, b)$.

Theorem 1.10 (Expected size of a $(\widetilde{W}, b)$-core) For $\widetilde{W}$ a simply-laced affine Weyl $\operatorname{group}, \operatorname{gcd}(h, b)=1$,

$$
\underset{\lambda \in \operatorname{core}(\widetilde{W}, b)}{\mathbb{E}}(\operatorname{size}(\lambda))=\frac{n(b-1)(h+b+1)}{24} .
$$

Theorem 1.11 (Variance of size on $(\widetilde{W}, b)$-cores) For $\widetilde{W}$ a simply-laced affine Weyl $\operatorname{group}, \operatorname{gcd}(h, b)=$ 1 ,

$$
\underset{\lambda \in \operatorname{core}(\widetilde{W}, b)}{\mathbb{V}}(\operatorname{size}(\lambda))=\frac{n h b(b-1)(h+b)(h+b+1)}{1440} .
$$

\subsection{Struture of the article}

The remainder of this paper is structured as follows. In Section 2 we review the basic notions of finite and affine Weyl groups. In Section 3, we review how $a$-cores fit into the framework of affine Weyl groups as the special case $\widetilde{W}=\widetilde{\mathfrak{S}}_{a} . \operatorname{In}$ Section 4 and Section 5 , we generalize simultaneous $(a, b)$-cores to $\widetilde{W}$ using the Sommers region $\mathcal{S}_{\Phi}(b)$, and we relate $\mathcal{S}_{\Phi}(b)$ and $b \mathcal{A}$. In Section 6, we generalize the statistic size to $\mathcal{S}_{\Phi}(b)$ for all affine Weyl groups In Section 7 we sketch the method by which Theorem 1.9, Theorem 1.10 and Theorem 1.11 are proven.

\section{Affine Weyl Groups}

In this section, we introduce finite and affine root systems (Sections 2.1 and 2.3) and associated data. We also define their associated hyperplane arrangements and Weyl groups [Section 2.2].

\subsection{Root Systems}

Let $\Phi$ be an irreducible crystallographic root system of rank $n$ with ambient space $V$. Define the root lattice $Q$ of $\Phi$ as the lattice in $V$ generated by $\Phi$. Let $\Phi^{+}$be a system of positive roots for it and let $\Delta=\left\{\alpha_{1}, \alpha_{2}, \ldots, \alpha_{n}\right\}$ be the corresponding system of simple roots. Then $\Phi$ is the disjoint union of $\Phi^{+}$ and $-\Phi^{+}$, and $\Delta$ is a basis for $V$.

For $\alpha \in \Phi$, we may write $\alpha$ in the basis of simple roots as $\alpha=\sum_{i=1}^{n} a_{i} \alpha_{i}$, where the coefficients $a_{i}$ are either all nonnegative or all nonpositive. We define the height of $\alpha$ as the sum of the coefficients: $\operatorname{ht}(\alpha):=\sum_{i=1}^{n} a_{i}$. Notice that $\operatorname{ht}(\alpha)>0$ if and only if $\alpha \in \Phi^{+}$and $\operatorname{ht}(\alpha)=1$ if and only if $\alpha \in \Delta$. 
There is a unique root $\tilde{\alpha}=\sum_{i=1}^{n} c_{i} \alpha_{i} \in \Phi$ of maximal height called the highest root of $\Phi$. We choose to normalize the inner product $\langle\cdot, \cdot\rangle$ on $V$ in such a way that $\|\tilde{\alpha}\|^{2}=2$. We define the Coxeter number of $\Phi$ as $h:=1+\operatorname{ht}(\tilde{\alpha})=1+\sum_{i=1}^{n} c_{i}$. Let $\rho:=\frac{1}{2} \sum_{\alpha \in \Phi^{+}} \alpha$. For a root $\alpha \in \Phi$, define its coroot as $\check{\alpha}:=\frac{2 \alpha}{\|\alpha\|^{2}}$. Define the dual root system of $\Phi$ as $\Phi^{\vee}:=\{\check{\alpha}: \alpha \in \Phi\}$. It is itself an irreducible crystallographic root system. We say that $\Phi$ is simply-laced if all roots $\alpha \in \Phi$ satsify $\|\alpha\|^{2}=2$. So in this case $\check{\alpha}=\alpha$ for all $\alpha \in \Phi$ and thus $\Phi=\Phi^{\vee}$. Define the coroot lattice $\check{Q}$ of $\Phi$ as the lattice in $V$ generated by $\Phi^{\vee}$. Let $\check{\rho}:=\frac{1}{2} \sum_{\alpha \in \Phi^{+}} \check{\alpha}$. Finally, let $\left(\check{\omega}_{1}, \check{\omega}_{2}, \ldots, \check{\omega}_{n}\right)$ be the basis that is dual to the basis $\left(\alpha_{1}, \alpha_{2}, \ldots, \alpha_{n}\right)$ of $V$ consisting of the simple roots, so that $\left\langle\breve{\omega}_{i}, \alpha_{j}\right\rangle=\delta_{i, j}$. Then $\check{\omega}_{1}, \check{\omega}_{2}, \ldots, \check{\omega}_{n}$ are the fundamental coweights. They are a basis of the coweight lattice $\check{\Lambda}:=\{x \in V:\langle x, \alpha\rangle \in \mathbb{Z}$ for all $\alpha \in \Phi\}$ of $\Phi$. We also have $\check{\rho}=\sum_{i=1}^{n} \check{\omega}_{i}$, so that $\langle\check{\rho}, \alpha\rangle=1$ for all $\alpha \in \Delta$ and thus $\langle\check{\rho}, \alpha\rangle=\operatorname{ht}(\alpha)$ for all $\alpha \in \Phi$.

We can write the highest root $\tilde{\alpha}$ (which is its own coroot) in terms of the coroots corresponding to the simple roots: $\tilde{\alpha}=\sum_{i=1}^{n} d_{i} \check{\alpha}_{i}$. Then we define the dual Coxeter number of $\Phi$ as $g:=1+\sum_{i=1}^{n} d_{i}$.

\subsection{Weyl Groups}

For $\alpha \in \Phi$ and $k \in \mathbb{Z}$, define the affine hyperplane $H_{\alpha}^{k}:=\{x \in V:\langle x, \alpha\rangle=k\}$ and let $s_{\alpha}^{k}: x \mapsto$ $x-\frac{2\langle x, \alpha\rangle-k}{\langle\alpha, \alpha\rangle}$ be the reflection through $H_{\alpha}^{k}$. We write $H_{\alpha}:=H_{\alpha}^{0}$ and $s_{\alpha}:=s_{\alpha}^{0}$.

Let $W$ be the group generated by $\left\{s_{\alpha}: \alpha \in \Phi\right\}$, called the Weyl group of $\Phi$. It acts on $\Phi$ and is minimally generated by the set $S:=\left\{s_{\alpha_{1}}, s_{\alpha_{2}}, \ldots, s_{\alpha_{n}}\right\}$ of simple reflections of $\Phi$. The Coxeter arrangement of $\Phi$ is the central hyperplane arrangement in $V$ given by all the hyperplanes $H_{\alpha}$ for $\alpha \in \Phi$. The complement $V \backslash\left\{H_{\alpha}\right\}_{\alpha \in \Phi}$ falls apart into connected components, which we call chambers. The Weyl group $W$ acts simply transitively on the set of chambers, so we define the dominant chamber

$$
C:=\{x \in V:\langle x, \alpha\rangle>0 \text { for all } \alpha \in \Delta\},
$$

and write any chamber as $w C$ for a unique $w \in W$.

Let $\widetilde{W}$ be the group generated by $\left\{s_{\alpha}^{k}: \alpha \in \Phi, k \in \mathbb{Z}\right\}$, called the affine Weyl group of $\Phi$. It is minimally generated by the set $\widetilde{S}:=S \cup\left\{s_{\tilde{\alpha}}^{1}\right\}$ of affine simple reflections of $\Phi$. So we may write any $\widetilde{w} \in \widetilde{W}$ as a word in the generators on $\widetilde{S}$. It is not hard to see that $\widetilde{W}$ acts on $\check{Q}$. To any $y \in V$, there is an associated translation $t_{y}(x):=x+y$. If we identify $\check{Q}$ with the corresponding group of translations acting on the affine space $V$, then we may write $\widetilde{W}=W \ltimes \check{Q}$ as a semidirect product.

The affine Coxeter arrangement of $\Phi$ is the affine hyperplane arrangement in $V$ given by all the affine hyperplanes $H_{\alpha}^{k}$ for $\alpha \in \Phi$ and $k \in \mathbb{Z}$. Its complement falls apart into connected components called alcoves. The affine Weyl group $\widetilde{W}$ acts simply transitively on the set of alcoves, so we define the (closed) fundamental alcove as

$$
\mathcal{A}:=\{x \in V:\langle x, \alpha\rangle \geq 0 \text { for all } \alpha \in \Delta \text { and }\langle x, \tilde{\alpha}\rangle \leq 1\}
$$

and write any alcove as $\widetilde{w} \mathcal{A}^{\circ}$ for a unique $\widetilde{w} \in \widetilde{W}$, where $\mathcal{A}^{\circ}$ is the interior of $\mathcal{A}$. We call $\widetilde{w}$ dominant if $\widetilde{w} \mathcal{A}^{\circ} \subseteq C$.

\subsection{Affine Root Systems}

We may also understand $\widetilde{W}$ in terms of its action on the set of affine roots $\widetilde{\Phi}$ of $\Phi$. To do this, let $\delta$ be a formal variable and define $\widetilde{V}:=V \oplus \mathbb{R} \delta$. Define the set of affine roots as $\tilde{\Phi}:=\{\alpha+k \delta:$ 
$\alpha \in \Phi$ and $k \in \mathbb{Z}\}$. If $\widetilde{w} \in \widetilde{W}$, write it as $\widetilde{w}=w t_{\mu}$ for unique $w \in W$ and $\mu \in \check{Q}$ and define $\widetilde{w}(\alpha+k \delta):=w(\alpha)+(k-\langle\mu, \alpha\rangle) \delta$. This defines an action of $\widetilde{W}$ on $\widetilde{\Phi}$. It imitates the action of $\widetilde{W}$ on the half-spaces of $V$ defined by the hyperplanes of the affine Coxeter arrangement. To see this, define the half-space $\mathcal{H}_{\alpha}^{k}:=\{x \in V:\langle x, \alpha\rangle>-k\}$. Then for $\widetilde{w} \in \widetilde{W}$ we have $\widetilde{w}\left(\mathcal{H}_{\alpha}^{k}\right)=\mathcal{H}_{\beta}^{l}$ if and only if $\widetilde{w}(\alpha+k \delta)=\beta+l \delta$. Define the set of positive affine roots as $\widetilde{\Phi}^{+}:=\left\{\alpha+k \delta: \alpha \in \Phi^{+}\right.$and $k \geq$ $0\} \cup\left\{\alpha+k \delta: \alpha \in-\Phi^{+}\right.$and $\left.k>0\right\}$, the set of affine roots corresponding to half-spaces that contain $\mathcal{A}^{\circ}$. So $\widetilde{\Phi}$ is the disjoint union of $\widetilde{\Phi}^{+}$and $-\widetilde{\Phi}^{+}$. Define the set of simple affine roots as $\widetilde{\Delta}:=\Delta \cup\{-\tilde{\alpha}+\delta\}$, the set of affine roots corresponding to half-spaces that contain $\mathcal{A}^{\circ}$ and share one of its defining inequalities. For $\widetilde{w} \in \widetilde{W}$, we say that $\alpha+k \delta \in \widetilde{\Phi}^{+}$is an inversion of $\widetilde{w}$ if $\widetilde{w}^{-1}(\alpha+k \delta) \in-\widetilde{\Phi}^{+}$, and we write

$$
\operatorname{inv}(\widetilde{w}):=\left\{\alpha+k \delta \in \widetilde{\Phi}^{+}: \widetilde{w}^{-1}(\alpha+k \delta) \in-\widetilde{\Phi}^{+}\right\}=\widetilde{\Phi}^{+} \cap \widetilde{w}\left(-\widetilde{\Phi}^{+}\right)
$$

as the set of inversions of $\widetilde{w}$.

Theorem 2.1 The positive affine root $\alpha+k \delta \in \widetilde{\Phi}^{+}$is an inversion of $\widetilde{w}$ if and only if the hyperplane $H_{\alpha}^{-k}$ separates $\widetilde{w} \mathcal{A}^{\circ}$ from $\mathcal{A}^{\circ}$.

Define the height of an affine root $\alpha+k \delta$ as ht $(\alpha+k \delta)=$ ht $(\alpha)+k h$. So ht $(\alpha+k \delta)>0$ if and only if $\alpha+k \delta \in \widetilde{\Phi}^{+}$and ht $(\alpha+k \delta)=1$ if and only if $\alpha+k \delta \in \widetilde{\Delta}$. For an integer $l$ with $-h<l<h$, let $\Phi_{l}$ be the set of roots in $\Phi$ of height $l$. Similarly, for any positive integer $b$, let $\widetilde{\Phi}_{b}$ be the set of affine roots in $\widetilde{\Phi}$ of height $b$. If we write $b=t h+r$ with $t, r \in \mathbb{Z}$ and $0 \leq r<h$, then $\widetilde{\Phi}_{b}=\{\alpha+t \delta: \alpha \in$ $\left.\Phi_{r}\right\} \cup\left\{\alpha+(t+1) \delta: \alpha \in \Phi_{r-h}\right\}$.

\subsection{Strange Identity}

To conclude this section, we recall the "strange formula" of H. Freudenthal and H. de Vries.

Theorem 2.2 Let $\Phi$ be an irreducible crystallographic root system of rank $n$. Let $h$ be the Coxeter number of of $\Phi, g$ the dual Coxeter number and recall that $\rho=\frac{1}{2} \sum_{\alpha \in \Phi^{+}} \alpha$. Then

$$
\frac{\|\rho\|^{2}}{2 g}=\frac{n(h+1)}{24} .
$$

The appearance of the number 24 in the denominator of the "strange formula" will explain its appearance in Theorems 1.9 and 1.10 .

\section{Cores}

In this section, we recall the bijection between $a$-cores and the minimal-length coset representatives for the parabolic quotient $\widetilde{\mathfrak{S}}_{a} / \mathfrak{S}_{a}$ Theorem 3.1. Using the isomorphism between $\widetilde{W} / W$ and $\check{Q}$ for $W=\mathfrak{S}_{a}$, we interpret and generalize cores as points in the coroot lattice $\check{Q}$.

\subsection{The Affine Symmetric Group and Cores}

The affine symmetric group has presentation

$$
\widetilde{\mathfrak{S}}_{a}:=\left\langle s_{0}, s_{1}, \ldots, s_{a-1}:\left(s_{i} s_{i+1}\right)^{3}=e,\left(s_{i} s_{j}\right)^{2}=e \text { if }|i-j|>1\right\rangle,
$$


where indices will always be taken modulo $a$. The elements $\widetilde{w} \in \widetilde{\mathfrak{S}}_{a}$ such that $\widetilde{w}^{-1} \mathcal{A}^{\circ} \subseteq C$ are the minimal-length right coset representatives for the parabolic quotient $\widetilde{\mathfrak{S}}_{a} / \mathfrak{S}_{a}$. By abuse of notation, we will associate elements of $\widetilde{\mathfrak{S}}_{a} / \mathfrak{S}_{a}$ with their minimal-length right coset representatives.

There is a bijection between $\widetilde{\mathfrak{S}}_{a} / \mathfrak{S}_{a}$ and $a$-cores, given as follows. Label the $(i, j)$ th box of the Ferrers diagram of an $a$-core $\lambda$ by its content $(j-i) \bmod a$. We define an action of $\widetilde{\mathfrak{S}}_{a}$ on the set of $a$-cores by defining how the simple reflections $s_{i}$ act. Given an $a$-core $\lambda$, we define $s_{i} \lambda$ (for $0 \leq i \leq a-1$ ) to be the unique $a$-core that differs from $\lambda$ only by boxes with content $i$. The partial order on core $(a)$ is given by letting $\lambda$ cover $\mu$ if and only if $\operatorname{size}(\lambda)>\operatorname{size}(\mu)$ and $\lambda=s_{i} \mu$ for some $i$.

Theorem 3.1 ([Las01]) The map

$$
\begin{aligned}
\operatorname{core}_{\mathfrak{S}}: \widetilde{\mathfrak{S}}_{a} / \mathfrak{S}_{a} & \rightarrow \operatorname{core}(a) \\
\widetilde{w} & \mapsto \widetilde{w} \cdot \emptyset
\end{aligned}
$$

is an isomophism between the weak order on $\widetilde{\mathfrak{S}}_{a} / \mathfrak{S}_{a}$ and the poset on core $(a)$ defined above.

Thus, $a$-cores are identified with elements of $\widetilde{\mathfrak{S}}_{a} / \mathfrak{S}_{a}$. Theorem 3.1 is illustrated in Figure 1 for $a=3$.

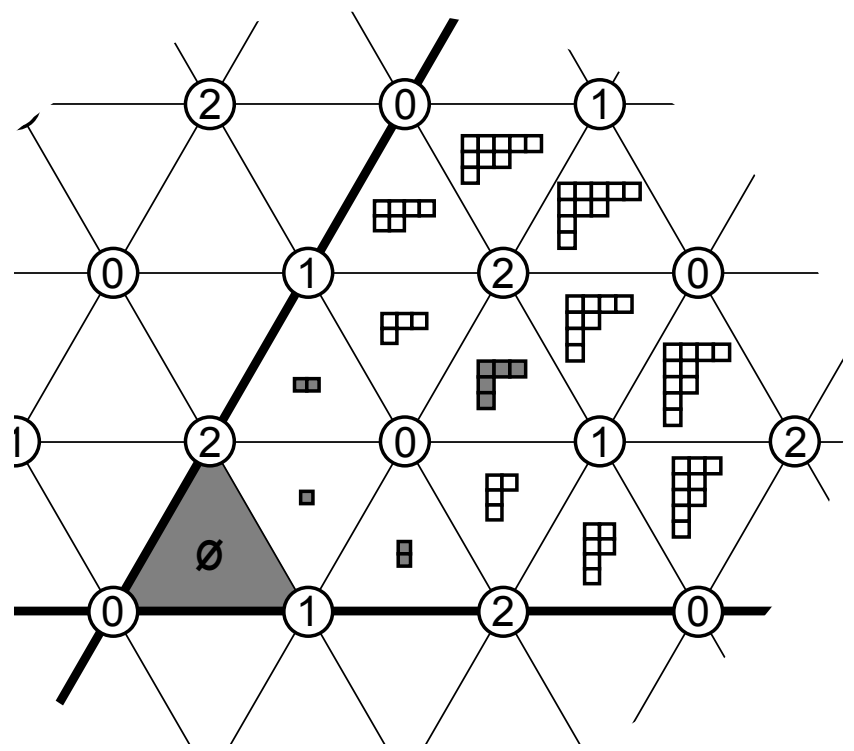

Fig. 1: The weak order on the minimal-length representatives $\widetilde{w} \in \widetilde{\mathfrak{S}}_{3} / \mathfrak{S}_{3}$ displayed as the dominant alcoves $\widetilde{w}^{-1} \mathcal{A}^{\circ}$ and the poset of 3 -cores. The five simultaneous $(3,4)$-cores are the empty core and the four cores shaded gray.

\subsection{Affine Weyl Groups and Cores}

There are two fundamentally different ways to think of $\widetilde{W}$. The first, mentioned in Section 2.2 is as $\widetilde{W}:=W \rtimes \check{Q}$, which we think of as tiling $V$ using bounded copies of $W$ centered at each point of the coroot lattice. The second way is as $\widetilde{W}:=(\widetilde{W} / W) \rtimes W$, which may be visualized as replicating a 
copy of the parabolic quotient $(\widetilde{W} / W)$ in each chamber of the Coxeter arrangement of $W$. These two constructions are related as follows.

Proposition 3.2 There is a canonical bijection

$$
\text { crt : } \begin{aligned}
(\widetilde{W} / W) & \rightarrow \check{Q} \\
w & \mapsto w(0) .
\end{aligned}
$$

Together with Theorem 3.1 this implies that the coroot points in $\widetilde{\mathfrak{S}}_{a}$ are in bijection with the set of $a$-cores. This suggests that the set of coroot points is the correct generalization of cores to any affine Weyl group $\widetilde{W}$. It is natural to write core $(\widetilde{W}):=\check{Q}$.

\section{Two Simplices}

In this section, we recall the definitions of two simplices associated to $\widetilde{W}$ Section 4. We see that they are equivalent up to an explicit rigid transformation (Theorem 4.2).

\subsection{Dilations of the Fundamental Alcove}

We write $b \mathcal{A}:=\{x \in V:\langle x, \alpha\rangle \geq 0$ for all $\alpha \in \Delta$ and $\langle x, \tilde{\alpha}\rangle \leq b\}$ for the $b$-fold dilation of the fundamental alcove, defined for any $b \in \mathbb{R}_{\geq 0}$. This region is bounded by the hyperplanes $\left\{H_{\alpha}: \alpha \in\right.$ $\Delta\} \cup\left\{H_{\tilde{\alpha}}^{b}\right\}$. Its volume is $b^{n}$ times that of the fundamental alcove $\mathcal{A}$, so it contains $b^{n}$ alcoves.

\subsection{Sommers Regions}

The second simplex, $\mathcal{S}_{\Phi}(b)$, is defined only for $b$ relatively prime to $h$. In this case write $b=t h+r$ with $t, r \in \mathbb{Z}_{\geq 0}$ and $0<r<h$. Define the Sommers region [Som05] as

$$
\mathcal{S}_{\Phi}(b):=\left\{x \in V:\langle x, \alpha\rangle \geq-t \text { for all } \alpha \in \Phi_{r} \text { and }\langle x, \alpha\rangle \leq t+1 \text { for all } \alpha \in \Phi_{h-r}\right\} .
$$

The significance of the Sommers region is as follows. Define $\widetilde{W}^{b}$ as the set of $\widetilde{w} \in \widetilde{W}$ that have no inversions of height $b$. So $\widetilde{W}^{b}=\left\{\widetilde{w} \in \widetilde{W}: \widetilde{w}^{-1}\left(\widetilde{\Phi}_{b}\right) \subseteq \widetilde{\Phi}^{+}\right\}$. By Theorem 2.1, we have that $\widetilde{w} \in \widetilde{W}^{b}$ if and only if none of the affine hyperplanes in

$$
\left\{H_{\alpha}^{-t}: \alpha \in \Phi_{r}\right\} \cup\left\{H_{\alpha}^{-t-1}: \alpha \in \Phi_{r-h}\right\}=\left\{H_{\alpha}^{-t}: \alpha \in \Phi_{r}\right\} \cup\left\{H_{\alpha}^{t+1}: \alpha \in \Phi_{h-r}\right\}
$$

separate $\widetilde{w} \mathcal{A}^{\circ}$ from $\mathcal{A}^{\circ}$. So $\widetilde{w} \in \widetilde{W}^{b}$ if and only if $\widetilde{w} \mathcal{A}^{\circ} \subseteq \mathcal{S}_{\Phi}(b)$.

\subsection{From $\mathcal{S}_{\Phi}(b)$ to $b \mathcal{A}$}

It turns out that $b \mathcal{A}$ and $\mathcal{S}_{\Phi}(b)$ are equivalent up to a rigid transformation, which may be realized as an element $\widetilde{w}_{b} \in \widetilde{W}$.

Theorem 4.1 For $b$ relatively prime to $h$, there exists a unique element $\widetilde{w}_{b} \in \widetilde{W}$ with

$$
b \frac{\check{\rho}}{h}=\widetilde{w}_{b}\left(\frac{\check{\rho}}{h}\right) .
$$

Theorem 4.2 The affine Weyl group element $\widetilde{w}_{b}$ maps $\mathcal{S}_{\Phi}(b)$ bijectively to $b \mathcal{A}$.

We stress that this bijection sends the set of coweight lattice points in $\mathcal{S}_{\Phi}(b)$ to the set of coweight lattice points in $b \mathcal{A}$, and similarly for coroot lattice points. 


\section{Simultaneous Cores}

Our generalization of the set of simultaneous $(a, b)$-cores to affine Weyl groups is the set of lattice points $\mathcal{S}_{\Phi}(b) \cap \check{Q}$ Definition 5.2). In Section 5.2, we recall M. Haiman's result on the number of such simultaneous cores (Theorem 1.8).

\subsection{Definition}

We now recall how to identify simultaneous $(a, b)$-cores using the bijection of Proposition 3.2 Let core $_{\check{Q}}:=\operatorname{core}_{\mathfrak{S}} \circ \mathrm{crt}^{-1}: \check{Q} \rightarrow \operatorname{core}(a)$.

Proposition 5.1 (|GMV, Joh15]) For $\operatorname{gcd}(a, b)=1$, core $(a, b)=\left\{\operatorname{core}_{\check{Q}}(\lambda): \lambda \in \mathcal{S}_{\Phi}(b) \cap \check{Q}\right\}$, where $\Phi$ is a root system for $\mathfrak{S}_{a}$.

We conclude that the coroot points in $\mathcal{S}_{\Phi}(b)$ generalize the set of simultaneous $(a, b)$-cores to all $\widetilde{W}$. We emphasize this with the following definition.

Definition 5.2 For $b$ relatively prime to $h$, we write core $(\widetilde{W}, b):=\mathcal{S}_{\Phi}(b) \cap \check{Q}$.

\subsection{Enumeration:Theorem 1.8}

Using Theorem 4.2, a result of Mark Haiman gives a nice product formula for the number of $(\widetilde{W}, b)$-cores.

Theorem 1.8 (Number of $(\widetilde{W}, b)$-cores; M. Haiman [Hai94, Theorem 7.4.4])

For $\operatorname{gcd}(h, b)=1$,

$$
|\operatorname{core}(\widetilde{W}, b)|=\frac{1}{|W|} \prod_{i=1}^{n}\left(b+e_{i}\right) .
$$

This number has since become known as the rational Catalan number associated to $W$ and $b$ (see, for example, [ARW13]).

\section{The Statistic size}

In this section, we interpret the statistic size, which counts the number of boxes in the Ferrers diagram of an $a$-core, in the language of root systems. We then extend this statistic to any affine Weyl group.

\section{1 size on Elements of $\widetilde{W}$}

Definition 6.1 For $\widetilde{w} \in \widetilde{W}$, define

$$
\operatorname{size}(\widetilde{w}):=\sum_{\alpha+k \delta \in \operatorname{inv}\left(\widetilde{w}^{-1}\right)} k .
$$

The statistic size is preserved under the bijection of Theorem 3.1

Proposition 6.2 The bijection core $\mathfrak{S}_{\text {from }} \widetilde{\mathfrak{S}}_{a} / \mathfrak{S}_{a}$ to core $(a)$ preserves size.

The statistic size of Definition 6.1 therefore generalizes size on $a$-cores to all elements of an affine Weyl group.

Example 6.3 The 3-core $\lambda=\square \square$ has 5 boxes. The corresponding $\widetilde{\mathfrak{S}}_{3}$-element $\widetilde{w}=s_{1} s_{2} s_{1} s_{0}-$ illustrated in Figure 1-has $\operatorname{size}(\widetilde{w})=5$ because the inversions of $\widetilde{w}^{-1}$ are the affine roots $-\tilde{\alpha}+1$. $\delta,-\alpha_{1}+1 \cdot \delta,-\alpha_{2}+1 \cdot \delta,-\tilde{\alpha}+2 \cdot \delta$, and $5=1+1+1+2$. 


\section{2 size as a Quadratic Form}

We can also view size as a statistic on $V$.

Definition 6.4 For any $x \in V$, define

$$
\operatorname{size}(x):=\frac{g}{2}\left\|x-\frac{\rho}{g}\right\|^{2}-\frac{g}{2}\left\|\frac{\rho}{g}\right\|^{2}=\frac{g}{2}\|x\|^{2}-\langle x, \rho\rangle .
$$

Note that by the "strange" formula Theorem 2.2, we have $\frac{g}{2}\left\|\frac{\rho}{g}\right\|^{2}=\frac{n(h+1)}{24}$.

Proposition 6.5 The bijection crt from $\widetilde{W} / W$ to $\check{Q}$ of Proposition 3.2 preserves size.

\section{Sketch of proofs of Theorems}

Interpreting core $(\widetilde{W}, b)$ as the set of lattice points in $\check{Q}$ in a simplex $\mathcal{S}_{\Phi}(b)$ and size as a quadratic form is the key to proving Theorem 1.9, Theorem 1.10 and Theorem 1.11. The first step is to translate the statistic size on $\mathcal{S}_{\Phi}(b)$ to a statistic zise $_{b}$ on $b \mathcal{A}$. Here it is necessary to use the assumption that $\widetilde{W}$ is simply-laced.

Definition 7.1 For any $x \in V$, define

$$
\operatorname{zise}_{b}(x):=\frac{g}{2}\left\|x-b \frac{\rho}{g}\right\|^{2}-\frac{g}{2}\left\|\frac{\rho}{g}\right\|^{2}
$$

Proposition 7.2 For $\widetilde{W}$ a simply-laced affine Weyl group with $\operatorname{gcd}(h, b)=1$, the affine Weyl group element $\widetilde{w}_{b}$ sends the statistic size on $\mathcal{S}_{\Phi}(b)$ to the statistic zise ${ }_{b}$ on $b \mathcal{A}$.

To prove Theorem 1.9 one just has to verify that 0 is the unique point in $\check{Q} \cap b \mathcal{A}$ of maximum zise ${ }_{b}$. Proving Theorem 1.10 and Theorem 1.11 requires results from Euler-Maclaurin theory. Let $\mathcal{P}$ be a $n$ dimensional rational convex polytope in a lattice $L$ (with generators a basis of $\mathbb{R}^{n}$ ), and let $p: \mathbb{R}^{n} \rightarrow \mathbb{R}$ be a polynomial of degree $r$. The weighted lattice-point enumerator for the bth dilate of $\mathcal{P}$ is

$$
\mathcal{P}_{p}^{L}(b):=\sum_{x \in b \mathcal{P} \cap L} p(x) .
$$

It turns out that $\mathcal{P}_{p}^{L}(b)$ is not only a quasipolynomial in $b$, but also satifies a reciprocity relation[ii)

Theorem 7.3 ([Bar06, BV07, BBDL ${ }^{+}$12], [AB14, Theorem 4.6]) For $\mathcal{P}, L$, and $p$ as above,

1. $\mathcal{P}_{p}^{L}(b)$ is a quasipolynomial in $b$ of degree $n+r$. Its period divides the least common multiple of the denominators of the coordinates (in the generators of $L$ ) of the vertices of $\mathcal{P}$.

2. If $\mathcal{P}^{o}$ is the interior of $\mathcal{P}$, then $\mathcal{P}_{p}^{L}(-b)=(-1)^{n}\left(-\mathcal{P}^{o}\right)_{p}^{L}(b)$.

\footnotetext{
(ii) Even though these results are well-known to the experts, it is difficult to find explicit statements in the literature that apply at once to rational polytopes and weighted lattice-point enumerators; see the remark in [Joh15. Section 1.2.2].
} 
From Proposition 7.2 and Theorem 7.3 we deduce that

$$
\sum_{\mu \in \mathscr{Q}^{\prime} \cap \mathcal{S}_{\Phi}(b)} \operatorname{size}(\mu)=\sum_{\mu \in \check{Q} \cap b \mathcal{A}} \operatorname{zise}_{b}(\mu)=\mathcal{A}_{\text {zise }}^{\check{Q}}(b)
$$

is a quasipolynomial in $b$ and determine its degree and period. Thus we can calculate it by evaluating it at a finite number of points. Unfortunately, this involves a case-by-case check. This allows us to determine

$$
\underset{\lambda \in \operatorname{core}(\widetilde{W}, b)}{\mathbb{E}}(\operatorname{size}(\lambda))=\frac{\mathcal{A}_{\text {zise }}^{\check{Q}}(b)}{\mathcal{A}_{1}^{\check{Q}}(b)}=\frac{n(b-1)(h+b+1)}{24},
$$

proving Theorem 1.10 A similar computation involving zise ${ }_{b}^{2}$ results in

$$
\underset{\lambda \in \operatorname{core}(\widetilde{W}, b)}{\mathbb{V}}(\operatorname{size}(\lambda))=\frac{n h b(b-1)(h+b)(h+b+1)}{1440},
$$

proving Theorem 1.11

\section{Acknowledgements}

We thank D. Armstrong for helpful conversations and encouragement and P. Johnson for clarifying certain details of his paper [Joh15]. The second author thanks D. Stanton for introducing him to cores, D. Armstrong and M. Beck for an interesting conversation, M. Guay-Paquet for clarifying the last equality of [Mac71, Theorem 8.16], and F. Saliola for the use of his hardware for certain computations.

\section{References}

[AB14] F. Ardila and E. Brugalle, The double Gromov-Witten invariants of Hirzebruch surfaces are piecewise polynomial, arXiv:1412.4563 (2014).

[Agg14] A. Aggarwal, Armstrong's conjecture for $(k, m k+1)$-core partitions, arXiv:1407.5134 (2014).

[AHJ14] D. Armstrong, C. Hanusa, and B. Jones, Results and conjectures on simultaneous core partitions, European Journal of Combinatorics 41 (2014), 205-220.

[And02] J. Anderson, Partitions which are simultaneously $t_{1}$-and $t_{2}$-core, Discrete Mathematics 248 (2002), no. 1, 237-243.

[Arm15a] D. Armstrong, Rational Catalan combinatorics, 2012 (accessed 12 May, 2015).

[Arm15b]_, personal communication, 2015.

[ARW13] D. Armstrong, B. Rhoades, and N. Williams, Rational associahedra and noncrossing partitions, The Electronic Journal of Combinatorics 20 (2013), no. 3, P54.

[Bar06] A. Barvinok, Computing the Ehrhart quasi-polynomial of a rational simplex, Mathematics of Computation 75 (2006), no. 255, 1449-1466. 
$\left[\mathrm{BBDL}^{+}{ }^{+} 2\right]$ V. Baldoni, N. Berline, J. De Loera, M. Köppe, and M. Vergne, Computation of the highest coefficients of weighted Ehrhart quasi-polynomials of rational polyhedra, Foundations of Computational Math. 12 (2012), no. 4, 435-469.

[BV07] N. Berline and M. Vergne, Local Euler-Maclaurin formula for polytopes, Mosc. Math. J 7 (2007), no. 3, 355-386.

[Fay11] M. Fayers, The t-core of an s-core, Journal of Combinatorial Theory, Series A 118 (2011), no. 5, 1525-1539.

[GKS90] F. Garvan, D. Kim, and D. Stanton, Cranks and t-cores, Inventiones Mathematicae 101 (1990), no. 1, 1-17.

[GMV] E. Gorsky, M. Mazin, and M. Vazirani, Affine permutations and rational slope parking functions, arXiv:1403.0303.

[Hai94] M. Haiman, Conjectures on the quotient ring by diagonal invariants, J. of Algebraic Combinat. 3 (1994), no. 1, 17-76.

[JK81] G. James and A. Kerber, The representation theory of the symmetric group, 1981.

[Joh15] P. Johnson, Lattice points and simultaneous core partitions, arXiv:1502.07934 (2015).

[Las01] A. Lascoux, Ordering the affine symmetric group, Algebraic combinat. and applications, Springer, 2001, pp. 219-231.

[Mac71] I. Macdonald, Affine root systems and Dedekind's $\eta$-function, Inventiones mathematicae 15 (1971), no. 2, 91-143.

[Nak40] T. Nakayama, On some modular properties of irreducible representations of a symmetric group, I, II, Japan. J. Math 17 (1940), 165-184.

[OS07] J. Olsson and D. Stanton, Block inclusions and cores of partitions, Aequationes math. 74 (2007), no. 1-2, 90-110.

[Som05] E. Sommers, b-stable ideals in the nilradical of a Borel subalgebra, Canadian math. bull. 48 (2005), no. 3, 460-472.

[Sut98] R. Suter, The number of lattice points in alcoves and the exponents of the finite Weyl groups, Mathematics of computation (1998), 751-758.

[SZ13] R. Stanley and F. Zanello, The Catalan case of Armstrong's conjecture on core partitions, arXiv:1312.4352 (2013).

[Van08] J. Vandehey, A general theory of $(s, t)$-core partitions, Ph.D. thesis, University of Oregon, 2008. 